\title{
Photon collider: a four-channel autoguider solution
}

John C. Hygelund, Rachel Haynes, Ben Burleson, Benjamin J. Fulton

John C. Hygelund, Rachel Haynes, Ben Burleson, Benjamin J. Fulton, "Photon collider: a four-channel autoguider solution," Proc. SPIE 7742, High Energy, Optical, and Infrared Detectors for Astronomy IV, 77421F (16 July 2010); doi: $10.1117 / 12.857761$

Event: SPIE Astronomical Telescopes + Instrumentation, 2010, San Diego, California, United States 


\title{
Photon Collider: A Four Channel Autoguider Solution
}

\author{
John C. Hygelund*, Rachel Haynes, Ben Burleson, Benjamin J. Fulton \\ Las Cumbres Observatory Global Telescope, 6740 Cortona Dr. Suite 102, Goleta, CA, USA 93117
}

\begin{abstract}
The "Photon Collider" uses a compact array of four off axis autoguider cameras positioned with independent filtering and focus. The photon collider is two way symmetric and robustly mounted with the off axis light crossing the science field which allows the compact single frame construction to have extremely small relative deflections between guide and science CCDs. The photon collider provides four independent guiding signals with a total of 15 square arc minutes of sky coverage. These signals allow for simultaneous altitude, azimuth, field rotation and focus guiding. Guide cameras read out without exposure overhead increasing the tracking cadence. The independent focus allows the photon collider to maintain in focus guide stars when the main science camera is taking defocused exposures as well as track for telescope focus changes. Independent filters allow auto guiding in the science camera wavelength bandpass. The four cameras are controlled with a custom web services interface from a single Linux based industrial PC, and the autoguider mechanism and telemetry is built around a uCLinux based Analog Devices BlackFin embedded microprocessor. Off axis light is corrected with a custom meniscus correcting lens. Guide CCDs are cooled with ethylene glycol with an advanced leak detection system. The photon collider was built for use on Las Cumbres Observatory's 2 meter Faulks telescopes and currently used to guide the alt-az mount.
\end{abstract}

Keywords: autoguider, relative deflections, CCD, leak detection, BlackFin, meniscus lens, cassegrain

\section{INTRODUCTION}

A ground up redesign of the back end instrumentation package for two-meter class altitude-azimuth Faulkes telescopes includes a four channel auto guider called the "Photon Collider". The Photon Collider has four independently focusing and filtering Santa Barbara Instrument Group (SBIG) auto guiding cameras rigidly coupled to the science Charged Coupled Device (CCD) providing four times the probability of finding a guide star compared to the single camera facility autoguider. The main science camera, Spectral, is a $4 \mathrm{k} \mathrm{x} 4 \mathrm{k}$ multi color imager. The cassegrain instrument package is comprised of the spectral camera with Cryotiger temperature control, shutter, custom designed filter wheel, and Photon Collider auto guider. The obvious advantage of the Photon Collider compared to the single camera facility auto guider is the increased sky coverage, but it also has the unique ability to track from four fields around the science image and provides guide feedback to the mount to properly track in not only altitude and azimuth, but also in cassegrain rotation. The multi signal feed back can also be used to better track focus by using one of the camera adjust to both sides of focus while the other cameras continue to track stars. The individual filters on the camera allow the guiding to be done in the same bandpass as the science camera, which preserves the relative placement of the stars as the telescope tracks in altitude. The relative change between the guide star location and the science star is a noticeable effect on long exposures without the use of the same filter. The guide cameras are right above the science camera bolted to the same casting and have minimal relative mechanical deflections between the guiders and the science camera. The optical design optimizes the size, location, and the quality of the guide star field with astigmatism correcting meniscus lenses. The cameras have thermo electric coolers that keep the CCD at consistent set point temperature, and the heat is removed from the instrument housing using glycol protected by a leak detection system. The camera control, motor drives, temperature monitoring, and leak monitor are control by custom designed Blackfin controller hosting a web interface.

*johnh@ @ lcogt.net; phone 1805880 1646; fax 1805961 1792; lcogt.net

High Energy, Optical, and Infrared Detectors for Astronomy IV, edited by Andrew D. Holland, David A. Dorn Proc. of SPIE Vol. $7742,77421 \mathrm{~F} \cdot$ ( ) 2010 SPIE · CCC code: 0277-786X/10/\$18 · doi: 10.1117/12.857761 


\section{METHODOLOGY}

\subsection{Optical Design}

The limiting aberration of the Faulkes telescopes at large field angles is astigmatism, inherent to their RitcheyChrétien design. For the larger field size of the spectral instrument, astigmatism correction was necessary. With a relatively simple meniscus lens, the astigmatism in the science field was reduced by approximately a factor of 10 . As shown in Figure 1, the autoguider pickoff mirrors come before the science field meniscus corrector. To mimic the presence of the appropriate off axis section of the meniscus in the autoguider field, a tilted plano-concave lens is used as a corrector. Due to the autoguider field being decentered in both $\mathrm{x}$ and $\mathrm{y}$ with respect to the science field, the corrector lens is tilted about two axes. The lens is secured with Epo Tec 301-2 epoxy in an invar optical mount. The optical design specifies an image footprint that is used to shape a series of knife edge baffles. The baffles are located at both the top and bottom of the instrument as well as the front side of each of the correcting lens and at the entrance to each of the guide cameras.

The nominal focus position of the camera is given $4.5 \mathrm{~mm}$ of back focus and $12.5 \mathrm{~mm}$ of front focus. The extra front focus allows the camera to remain in focus when the science camera is purposely defocused.

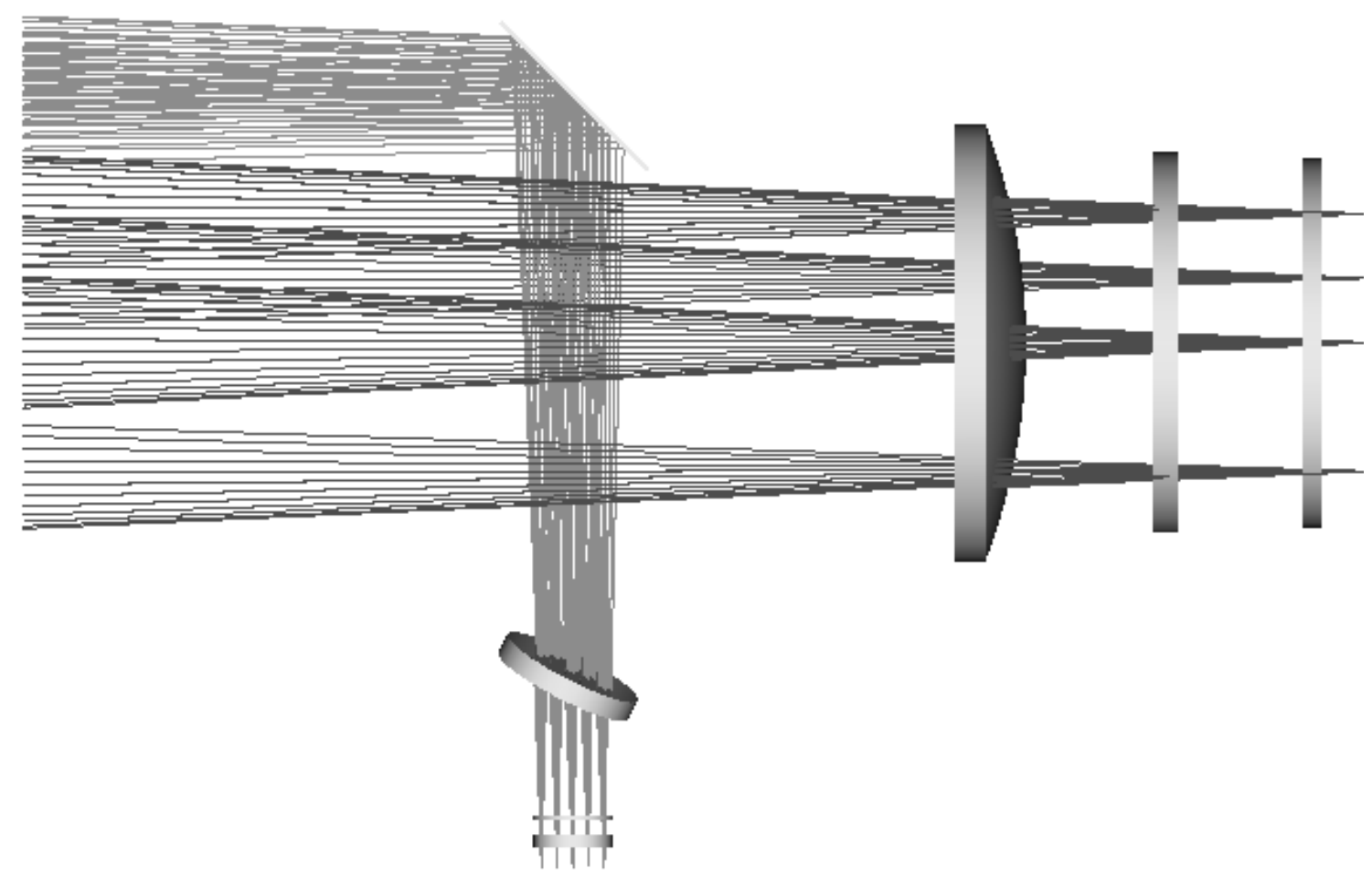

Figure 1. Optical layout of the Spectral instrument. The unfolded configuration is the science field, shown with meniscus corrector. The folded configuration shows one of the four autoguiders. The plane parallel plates shown in both configurations are filters and camera windows. 

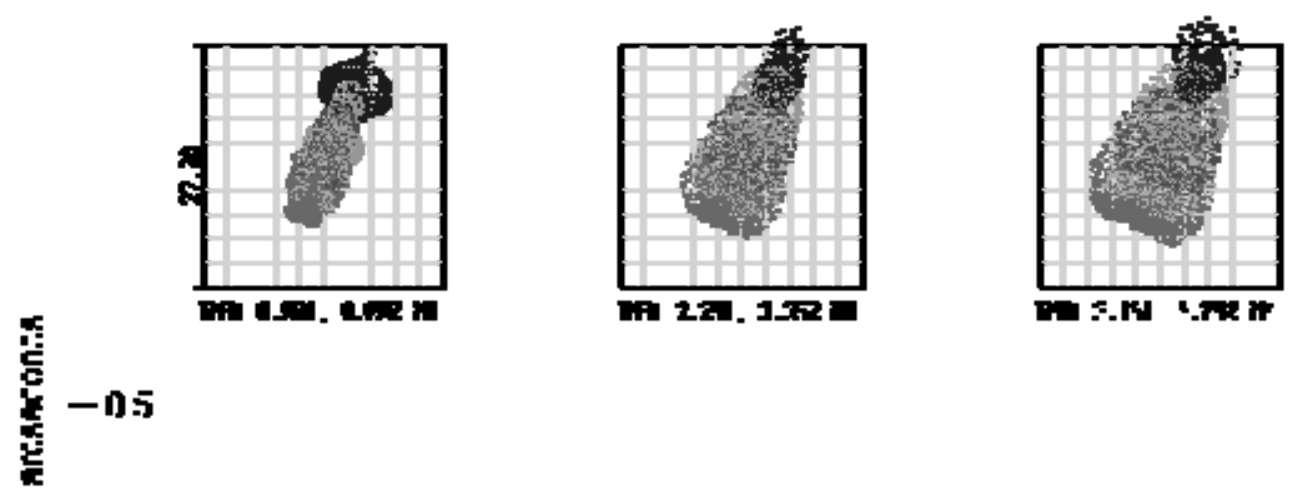

ด.

0.6oks

0.75er

0.07EO
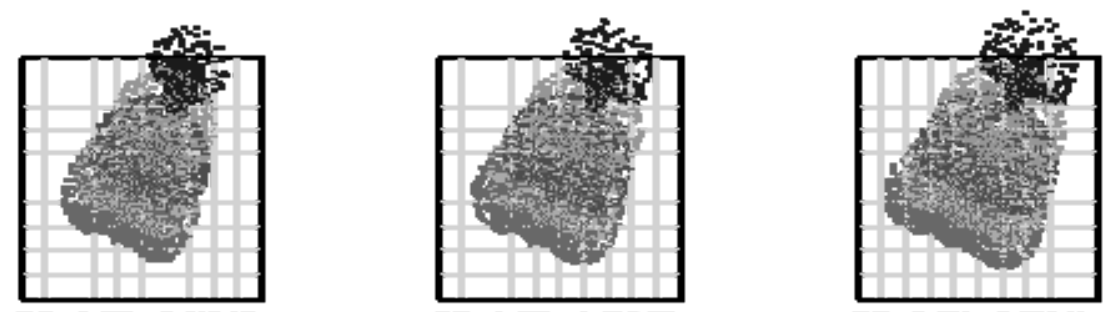

Ti. ‘.․ 4.ก7

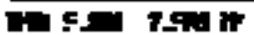

\section{$-1.0$}

Figure 2. Spot Diagrams for autoguider. Box size is $27 \mathrm{um}$, equivalent to $3 \mathrm{x} 3$ binning of 9um pixels. Wavelengths drawn are $450-850 \mathrm{~nm}$.

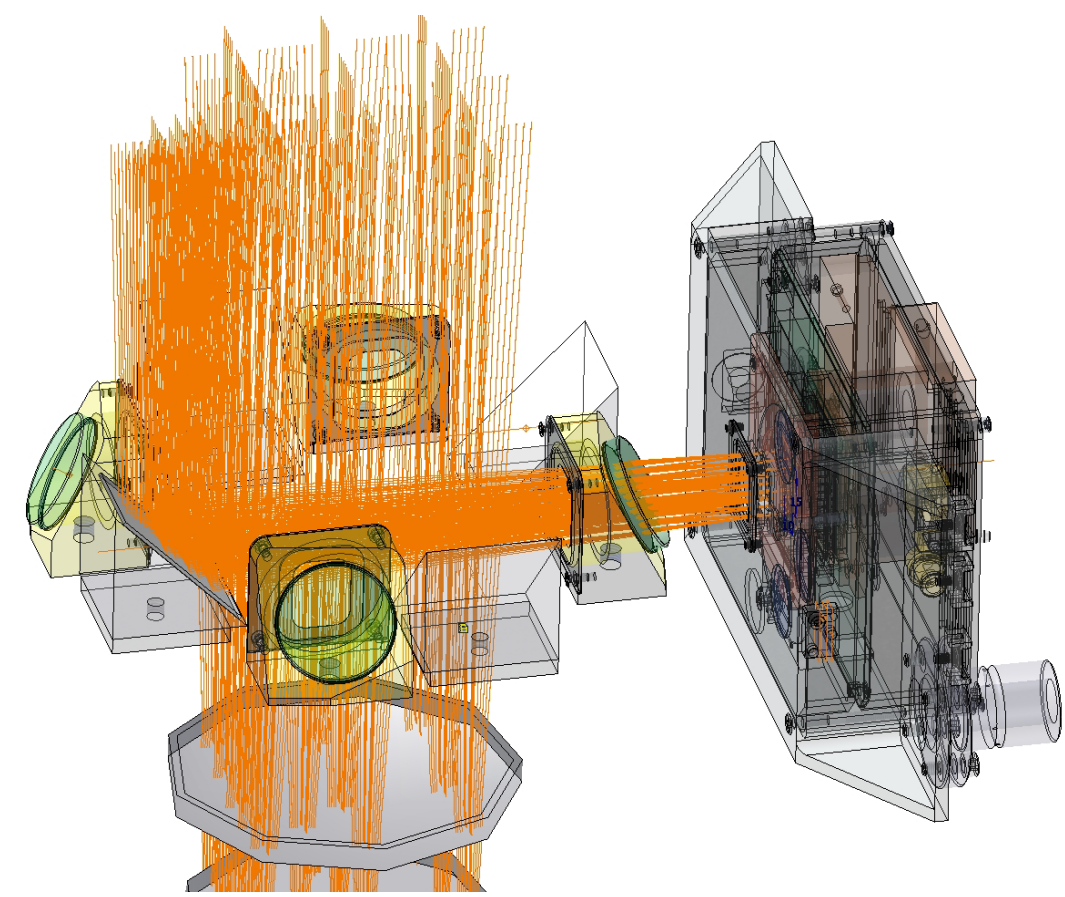

Figure 3. Light ray trace of one of the autoguider cameras showing the pick off mirror sending the light thru the meniscus lens onto the camera CCD. 


\subsection{Mechanical Design}

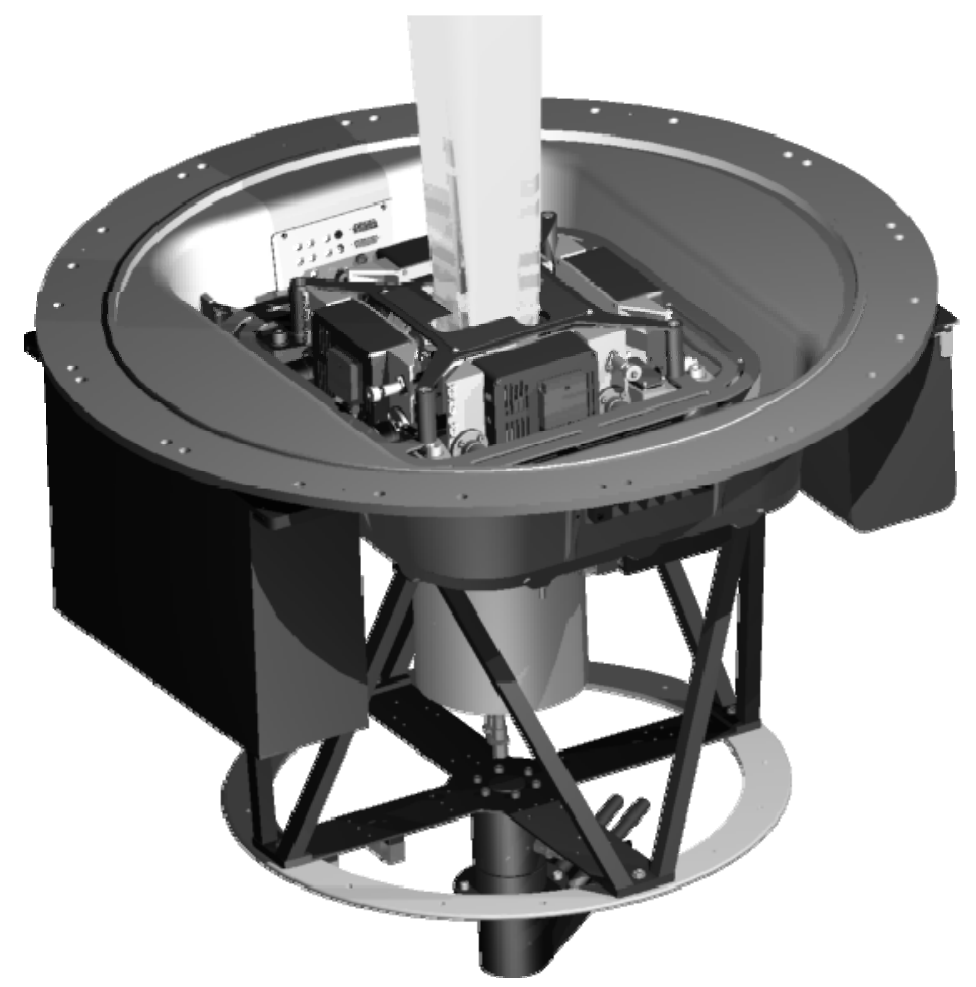

Figure 4. The Photon Collider inside the casting showing the Spectral camera on the bottom surrounded by the blue safety cage. Light from the telescope is shown in yellow. Various computers and other instrument equipment are shown bolted on the underside of the casting.

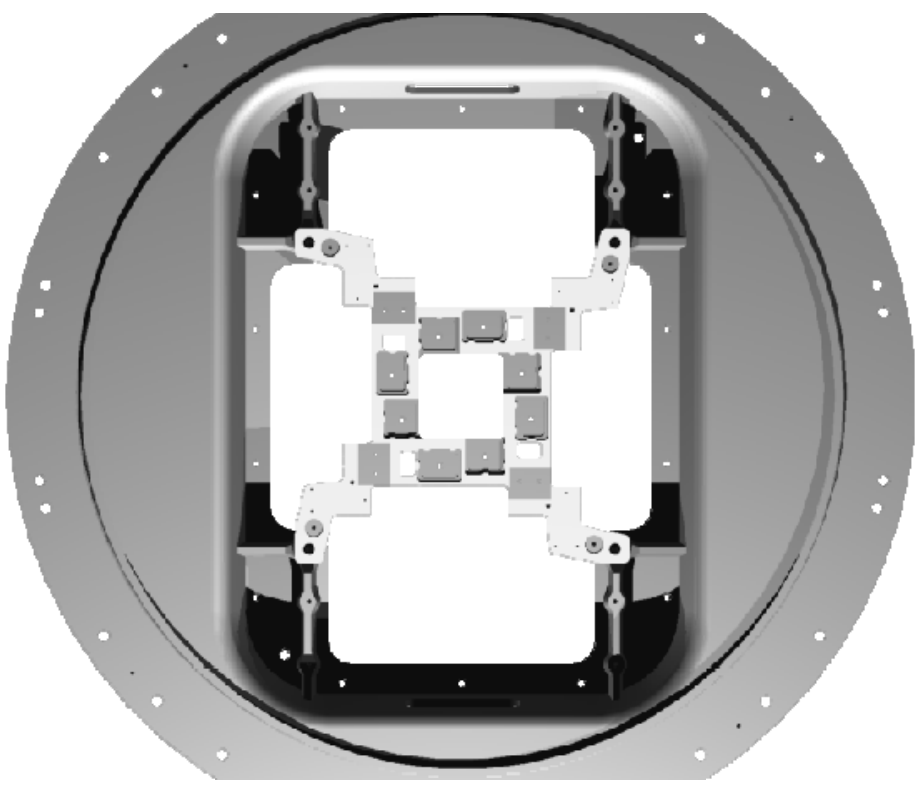

Figure 5. Photon Collider frame nested in the cast instrument housing. 
The Photon Collider has a simple four-point attachment interface inside a cast instrument housing, shown in Figure 5, for ease of access and removal. The two way symmetric off axis mechanical design couples the auto guider to the science CCD by sending the light across the science field allowing the camera to be placed closer to the science field and more robustly supported. If oriented with pickoff mirrors the other direction and the guider light directed away from the science field the camera must be supported further away from the science camera increasing the relative deflections. The location of the Photon Collider closer in to the field and directly atop of the science camera is inherently stiffer and more robust than the facility autoguider cantilevered off one of the ports on the acquisition and guider box. The two-way symmetric design gives each of the cameras the similar deflection characteristics independent of the gravity vector. The design criteria for the relative deflection between the autoguider CCD and the science CCD was 2 um over any 15 minute exposure and 20um over the all accessible gravity vectors. Finite Element Analysis (FEA) was used to analyze the mechanical structure to achieve the design criteria. The unique cross-field pickoff design demands a dense compact design. Figure 6 shows the densely packed design that houses four guide cameras with accompanying bearings and motors in a roughly 13 " square package no taller than the guide cameras.
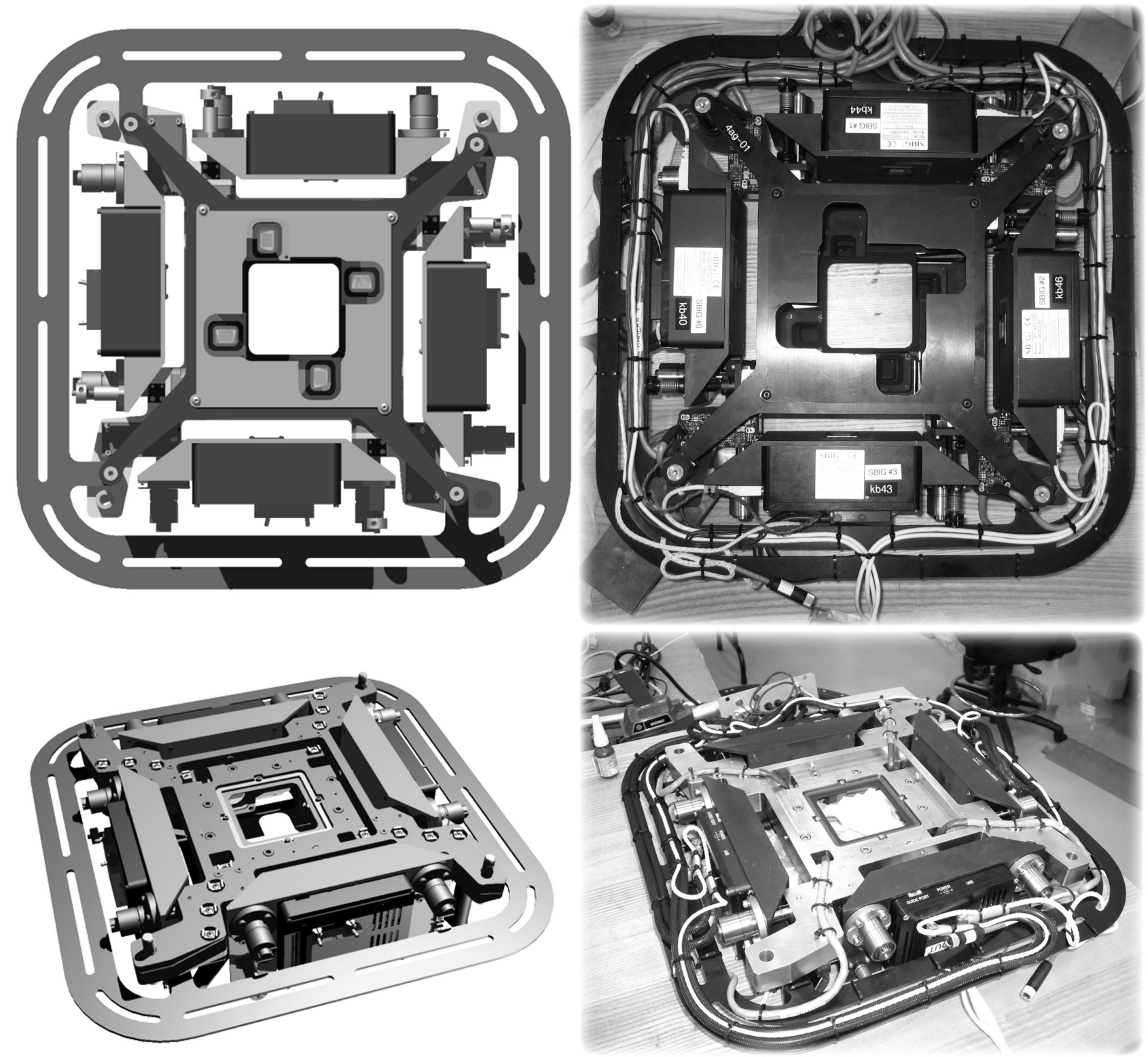

Figure 6. Photon Collider shown with 3D Computer Aided Drafting (CAD) and as built in top and bottom views. 
The frame is made from 300 series stainless steel for corrosion resistance and (Coefficient of Thermal Expansion) CTE matching to the instrument casting and invar optical mounts. The frame positions the optical elements of the design as well as four sets of corebored THK splined linear bearings. The linear bearings support the SBIG cameras positioned along a total of $17 \mathrm{~mm}$ of focus travel for each of the auto guider cameras.

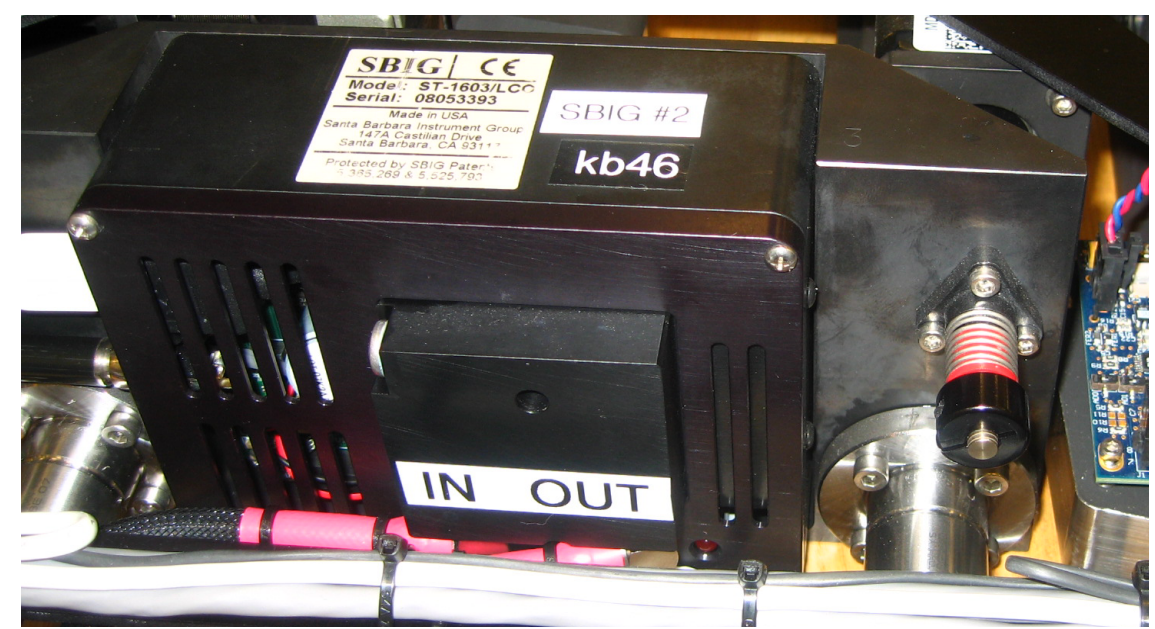

Figure 7. The back of the SBIG autoguider camera showing the glycol manifold, linear bearings, and the leadscrew.

Camera focus drive is provided by Kerk $1 \mathrm{~mm}$ lead anti backlash lead screw with Kerkote TFE coating. The lead screw torque is provided by IMS M-Drive 17 motor with 5 microns of focus per (non micro) step of the motor. The micro stepping is turned off so that any hold current heat generation is avoided. Limits are handled by hall effect sensors directly mount to pcb boards that sense magnets mounted on the camera housing. The repeatability of the hall effect sensors are +/- $2.5 \mathrm{um}$. Magnets are positioned with an adjustable mount on the side of the camera for calibrating an equal home position on each of the cameras. Hard stops on both directions of travel safely limit motion in case of software failure. Optical components are glued on to invar and stainless mounts and are bolted in for easy of removal for cleaning or replacement.

\subsection{Thermal Control}

The SBIG model ST-1603ME CCDs are cooled with ethylene glycol and an advanced leak detection system. The glycol system is pumped from a Thermo Cube in parallel with other instruments. The set point for the glycol temperate is monitored and kept above dew point to avoid any condensation on the cameras or glycol lines.

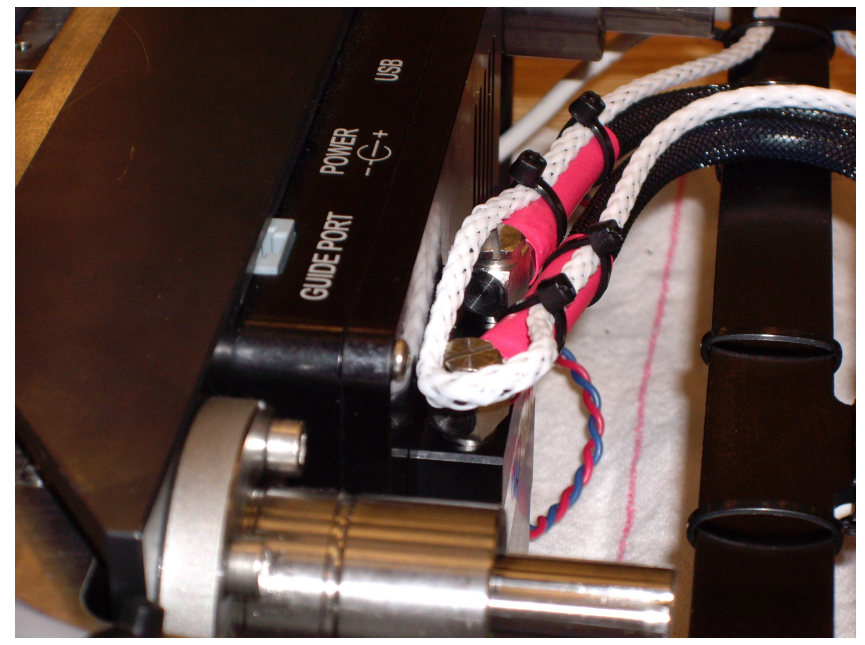

Figure 8. The AT monitor leak detection cable routed along the glycol tubing and over the stainless Beswick fluid fittings (shown upside down). 
The basis of the leak detection system is an AT Monitor leak detection cable that is made of a nylon wicking outer casing that bounds two individually wrapped stainless steel electrodes. In the presence of water or other conducting liquid the electrodes are shorted. The electrical short is monitored by the leak detector control box that closes a solenoid on the supply side of the glycol manifold and flips a relay state to alert the computer via blackfin. The passive one way valve prevents glycol from back flowing into the spectral casting. The "wet" state monitored by the computer notifies via email. The leak detection cable is run over the all the internal fittings and the length of the glycol tubing that is inside the casting. Leak detection cable is also run in the bottom of the casting where liquid would pool. The glycol tubing is medical grade peristaltic pump tubing that is rated for continuous flex and coated with expandable nylon casing for abrasion resistance. The lines are routed continuously from the camera outside the casting to minimize number of fittings in the casting.

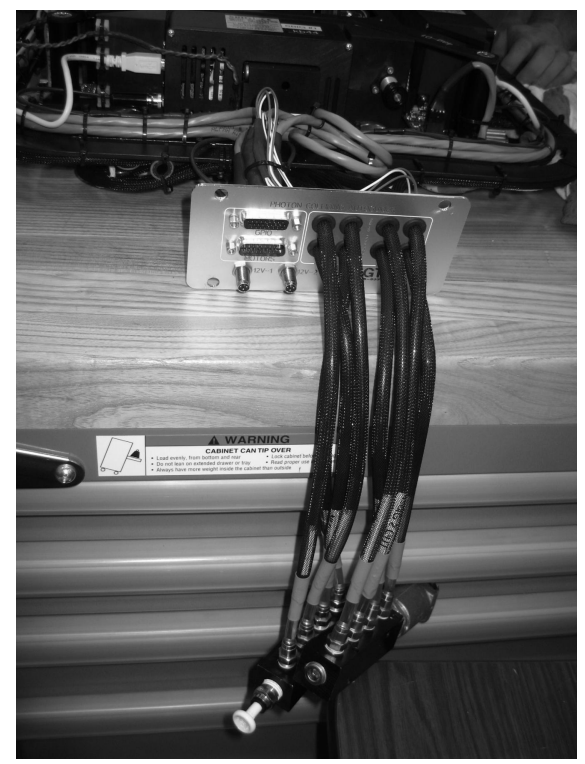

Figure 9. Glycol tubing run continuously from the camera to the tubing manifolds that is normally mounted on the outside of the instrument casting.

For bench testing the system glycol temperature was set below the dew point. Condensation first accumulated on the back of the camera enough to drip onto the water sensing cable below. The 'leak' was detecting and flow was diverted from the cameras until the system came back to temperature and the dew evaporated, at which point the circuit opened and flow began again. This process repeated 10 times over the course of the night with each wetness event showing in the status reported by the blackfin.

\subsection{Electrical Design}

The four cameras are controlled with a custom web services interface from a single Linux based industrial PC, and the autoguider mechanism and telemetry is built around a uCLinux based Analog Devices Blackfin embedded microprocessor. The Blackfin will run the focus motors and manage limits, temperature sensing, and leak detection.

The Blackfin is flashed with U-Boot bootloader. On the first boot, U-Boot will download a compressed uClinux image (uImage) and save the image to flash memory. On subsequent reboots, U-Boot will automatically boot the saved uImage. Part of the uClinux boot process looks for device application software updates (also stored in flash memory) and installs the updates if they exist. Device application software updates are downloaded and saved to flash manually via a script. This scheme allows the Blackfin to boot without network connectivity as well as support software updates.

The Blackfin controller runs uClinux which runs Boa webserver, allowing simple web-based control of the device. Standard HTTP GET and POST methods are used to query status and command the device. Application software for the 
Photon Collider is written in C and uses Boa's CGI. The standard HTTP interface provides an engineering test environment in any web browser. No software changes are needed to move from the test environment to production.

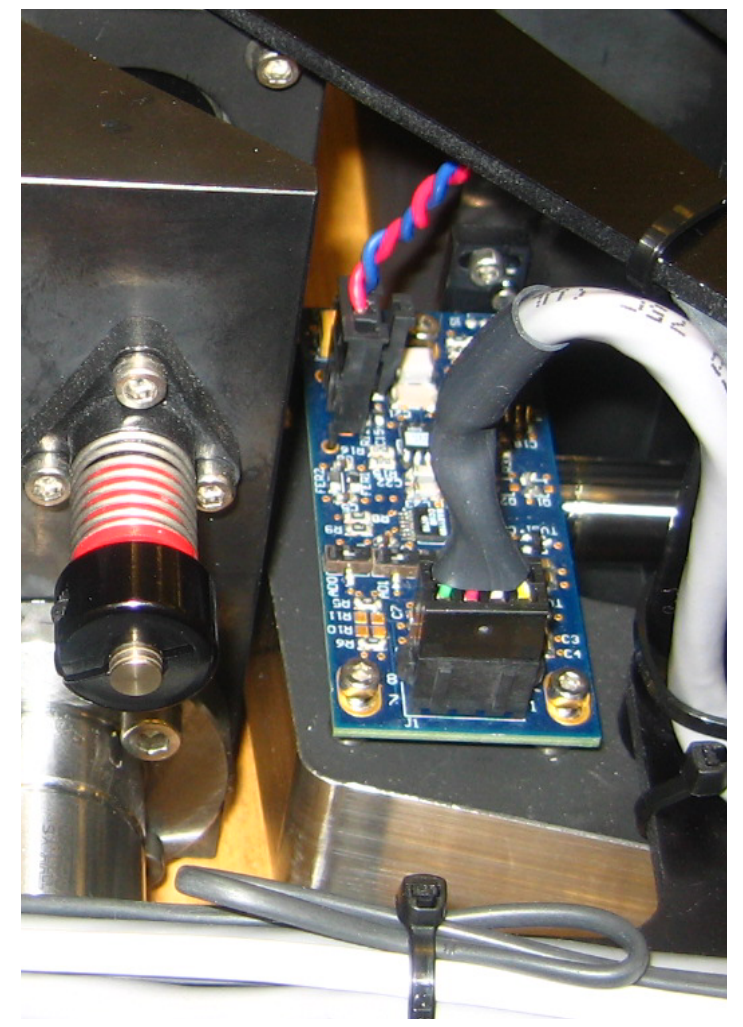

Figure 10. GPIO board for limit sensing and temperature monitoring.

Most hardware interfaces utilize the Blackfin's General Purpose Input Output (GPIO). This includes proximity sensors, wetness sensor, and motor control. The Blackfin's I2C bus is used to query ADCs for temperature monitoring. Stepper motors are controlled by three GPIO lines per motor (enable, direction, and step). An algorithm was written to control the acceleration and deceleration of the motors. The focus motor moves the camera along an axis parallel to the light beam. The focus of the four cameras is controlled via the web interface, and each camera can be focused independently. Two of the temperature sensors are thermally glued into the Photon Collider frame, and the remaining two sensors are attached to the glycol manifold on the back of the camera for a redundant monitoring of camera temperature.

\section{DATA AND RESULTS}

Currently the Photon Collider only provides guide corrections to the telescope from one autoguider at a time during the automated science control of the telescope. The control software still need to be developed to analyze and combine signals from more than one auto guider at a time. The telescope control software also doesn't allow for more than one autoguider to take images automatically. The guiders can be manually triggered, and this function has been used for the test results below. Results for the on sky appearance, performance of the autoguiders, and proposed multi channel logic are provided below. 


\subsection{On Sky Appearance}

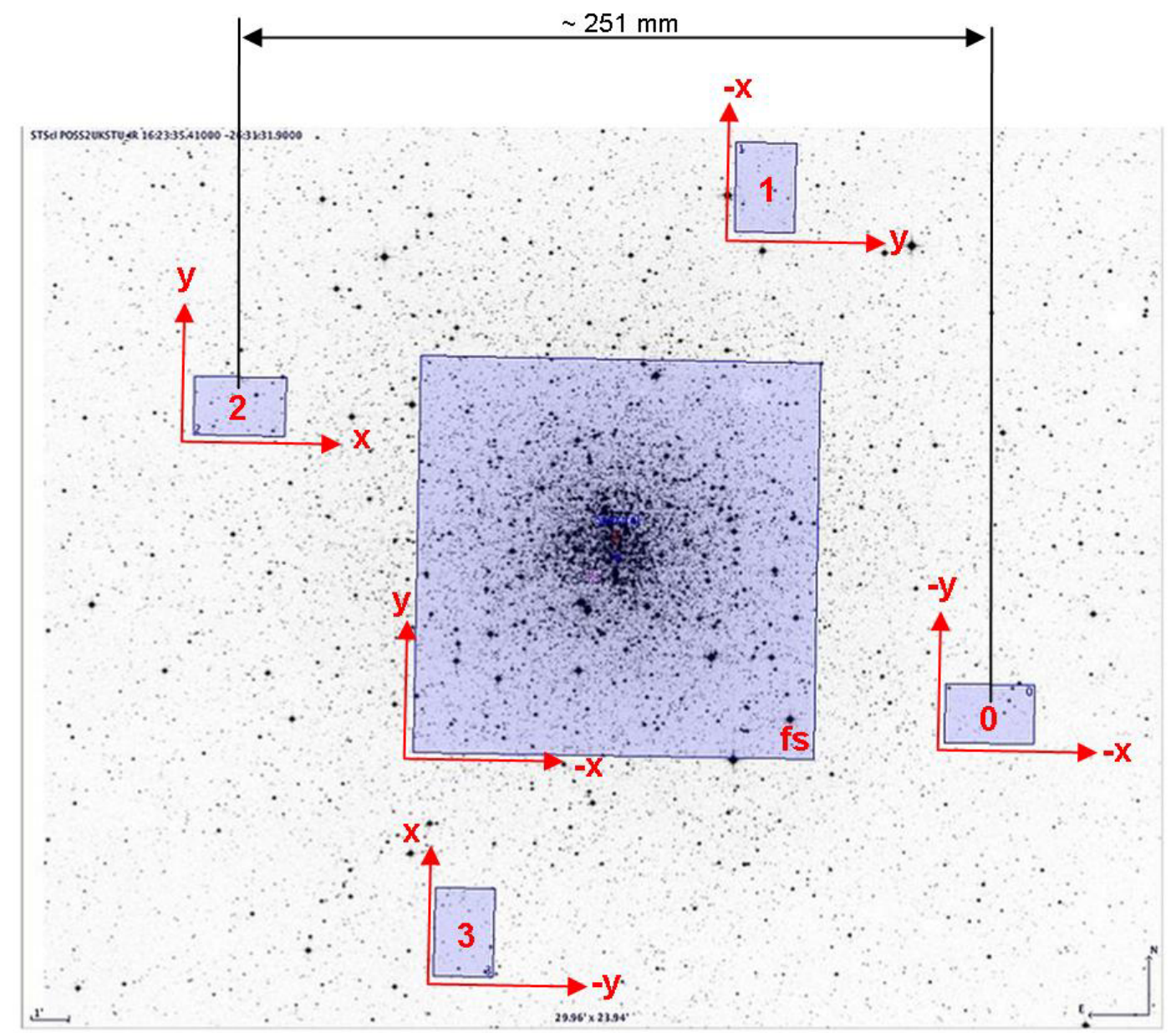

Figure 11. On sky appearance of the autoguiders with relative size to the science camera. The orientations of the guide chips shows how the orientations of the chips can be transformed to match the science frame. An approximate distance is shown for scale.

Advanced World Coordinate System (WCS) fits were used to find precise locations of the guide chips along with the precise pixel scales of each of the autoguiders. The relative locations are shown with the $\mathrm{x}$ and $\mathrm{y}$ transformations for each of the cameras relative to the main science field.

\subsection{Autoguider Performance}

The Faulkes telescope in Maui was used to collect some initial data from one of installed Photon Colliders. A dense star field was chosen for an hour long unguided image series. Due to the lack of control software development, all four of the autoguiders were manfully exposed in time with the science camera for the duration of the experiment. The data provided 30 data points at about two minute intervals of $\mathrm{x}$ and $\mathrm{y}$ centroid data for the science camera and all four of the autoguider cameras. All the images were analyzed for centroid shift using a World Coordinate System (WCS) fit. Each of the autoguiders had multiple stars. The analysis of the data is shown in the results below. 


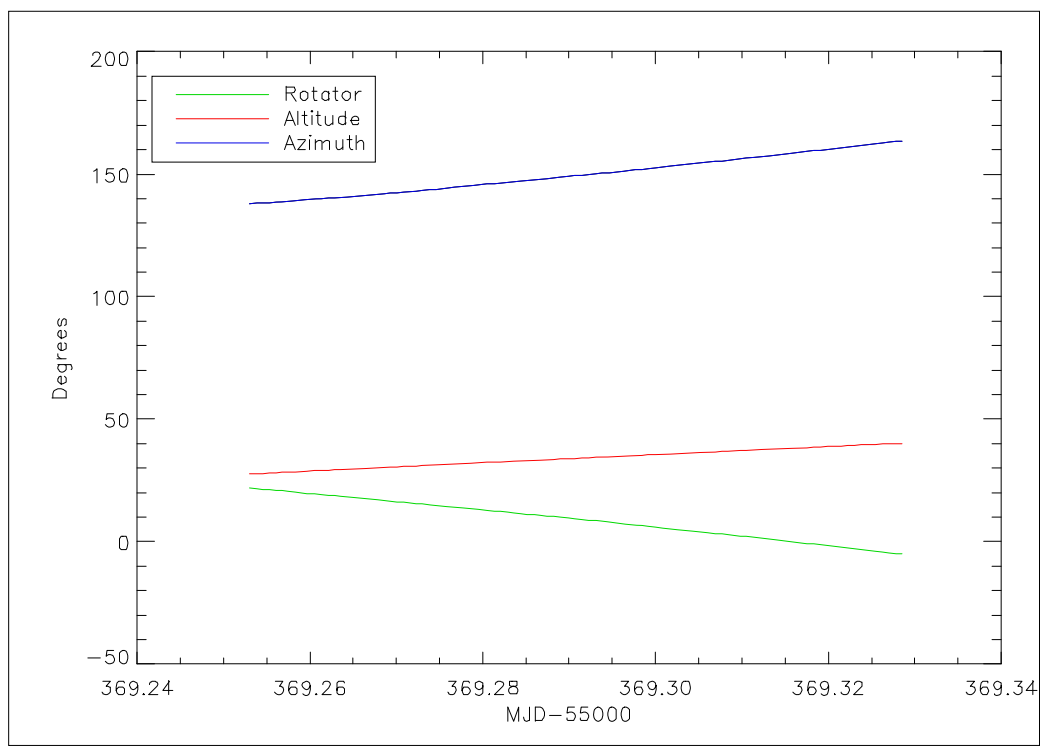

Figure 12. Telescope mount position for the duration of testing image series.
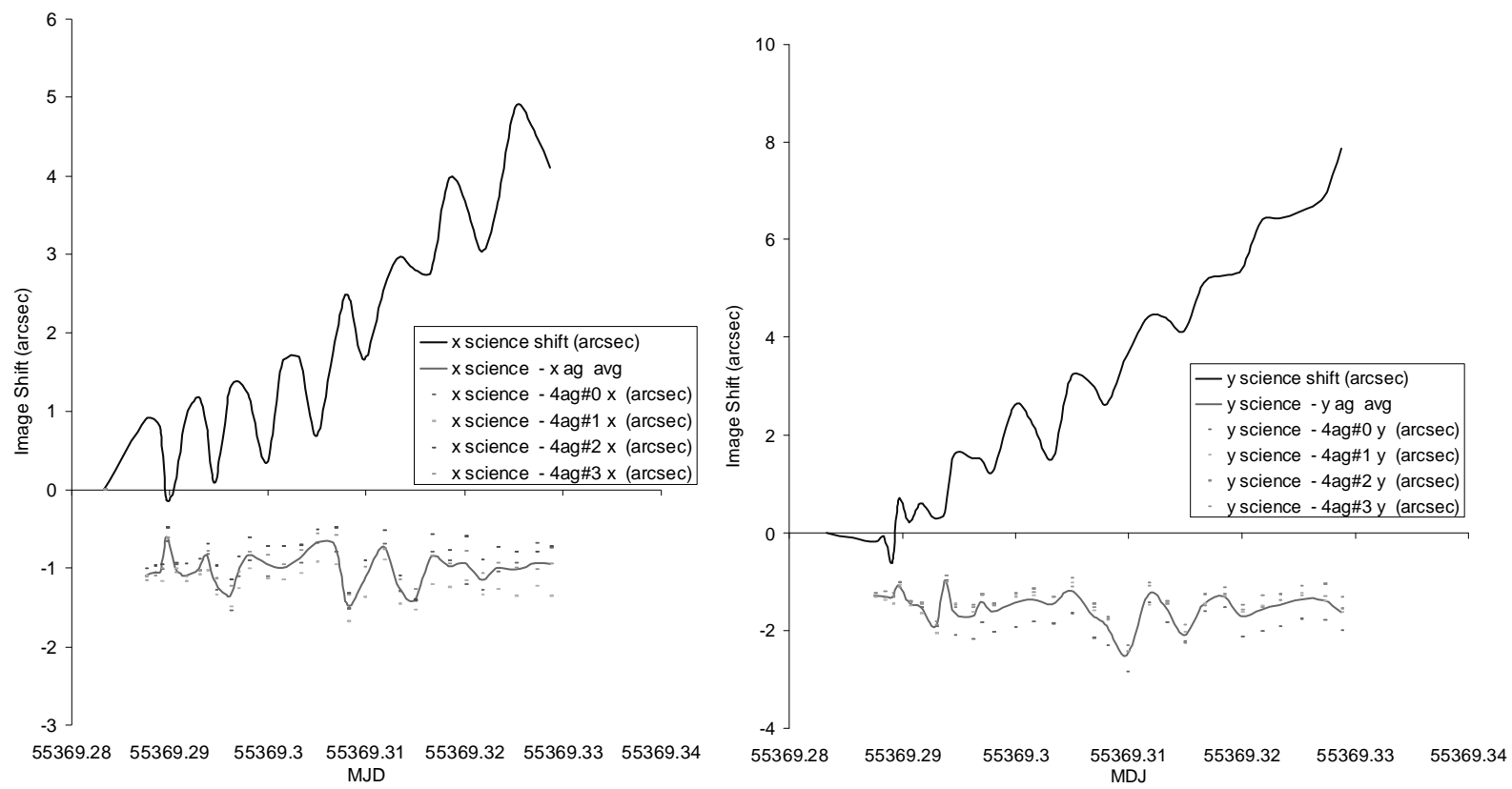

Figure 13. Drift of the science camera in both $\mathrm{x}$ and $\mathrm{y}$ directions, and calculated corrected drift of the science camera.

The unguided tracking shows a positive drift in both $\mathrm{x}$ and $\mathrm{y}$. The drift of each autoguider and the average of all four of the guider drifts are individually subtracted from the science drift resulting in a calculated "guided" performance. The pink line shows what the theoretical tracking would have been if tracking had been turned on for the image series. Each of the data points surrounding the pink average line are the theoretical tracking using only one of the guide cameras. The average is a marginally better tracking than any of the individual guide cameras. This averaging probably only benefits by averaging seeing effects from the four guide cameras, which would be more beneficial on short guide exposures. The guide exposures for this experiment were $40 \mathrm{sec}$, but the autoguider is normally run with ten second exposures. The tracking from any individual guide camera and the average of all four is $+/-.5 \mathrm{arc} \mathrm{sec}$. The average of the for camera will 
be a preferred guide correction if all the guiders have stars and are not being used for focus tracking. If only one guider has a star the $\mathrm{x}, \mathrm{y}$ corrections will still be very good.
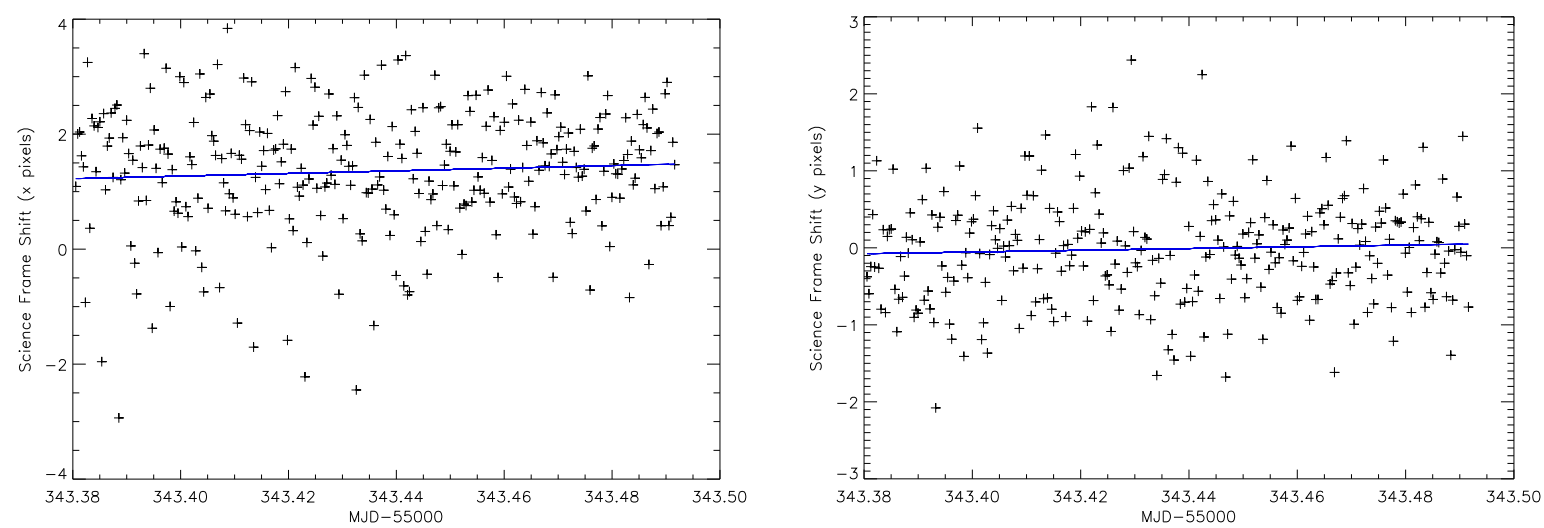

Figure 14. 3+ hour guided exposure with only autoguider (\#0)

Figure 14 shows the $\mathrm{x}$ and $\mathrm{y}$ drifts on the science camera for a 3+ hour actively guided exposure series. The camera was binned 2x2 (.304 pixel/arcsec) showing less than an arcsecond of scatter in the pointing. The drift over the length of the exposure is negligible and the slope of the blue fit line is within the error of the fit, meaning that the autoguider performs as expected without long term drift. The scatter corresponds with the theoretical calculated autoguide performance from Figure 13. The performance seen in Figure 14 was with 15 second guiding frequency, and the calculated performance from Figure 13 is with 60 second guiding frequency. This explains the slightly better scatter seen in the actual guided exposures.

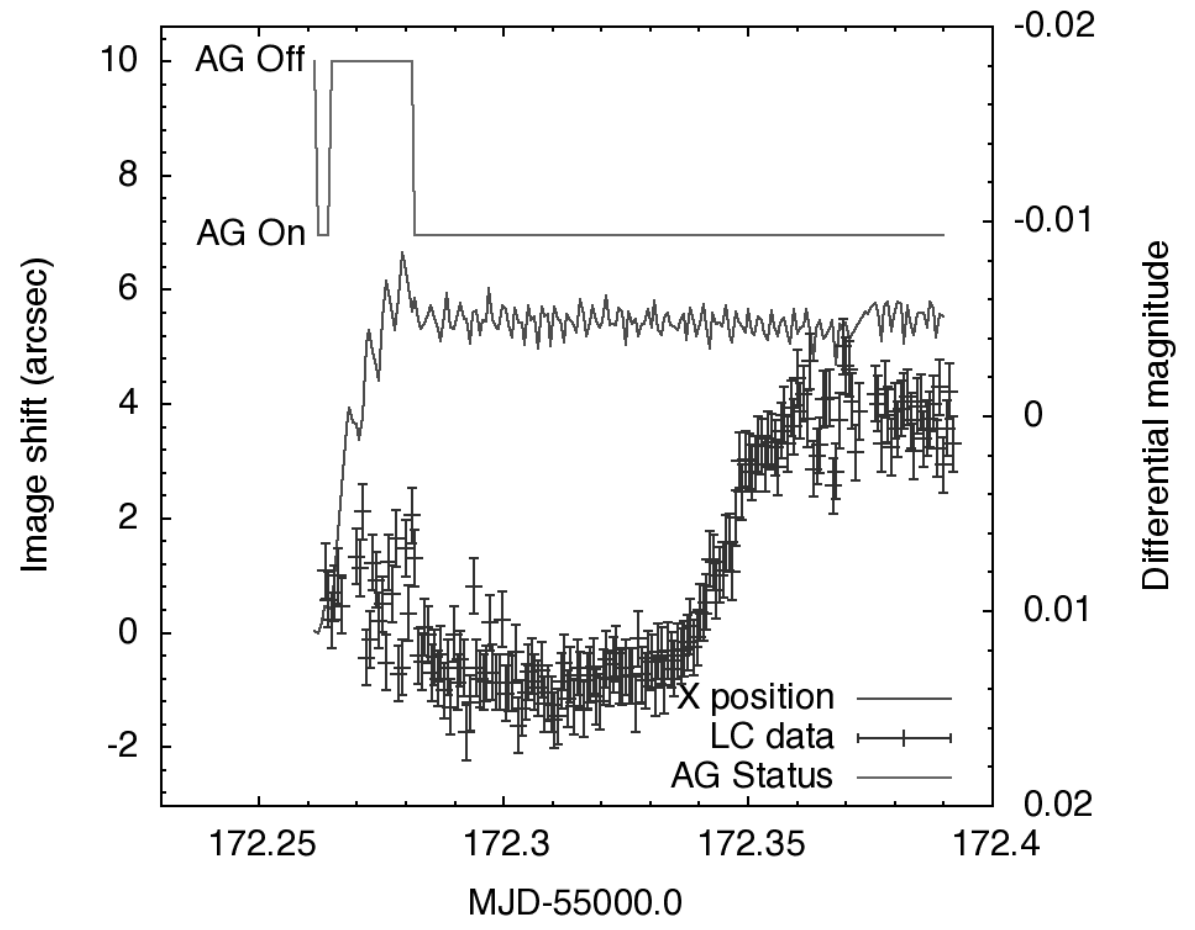

Figure 15. Partial planet transit showing improvement in the light curve scatter and stability when the autoguider is tracking. 


\subsection{Multi Channel Feedback Analysis}

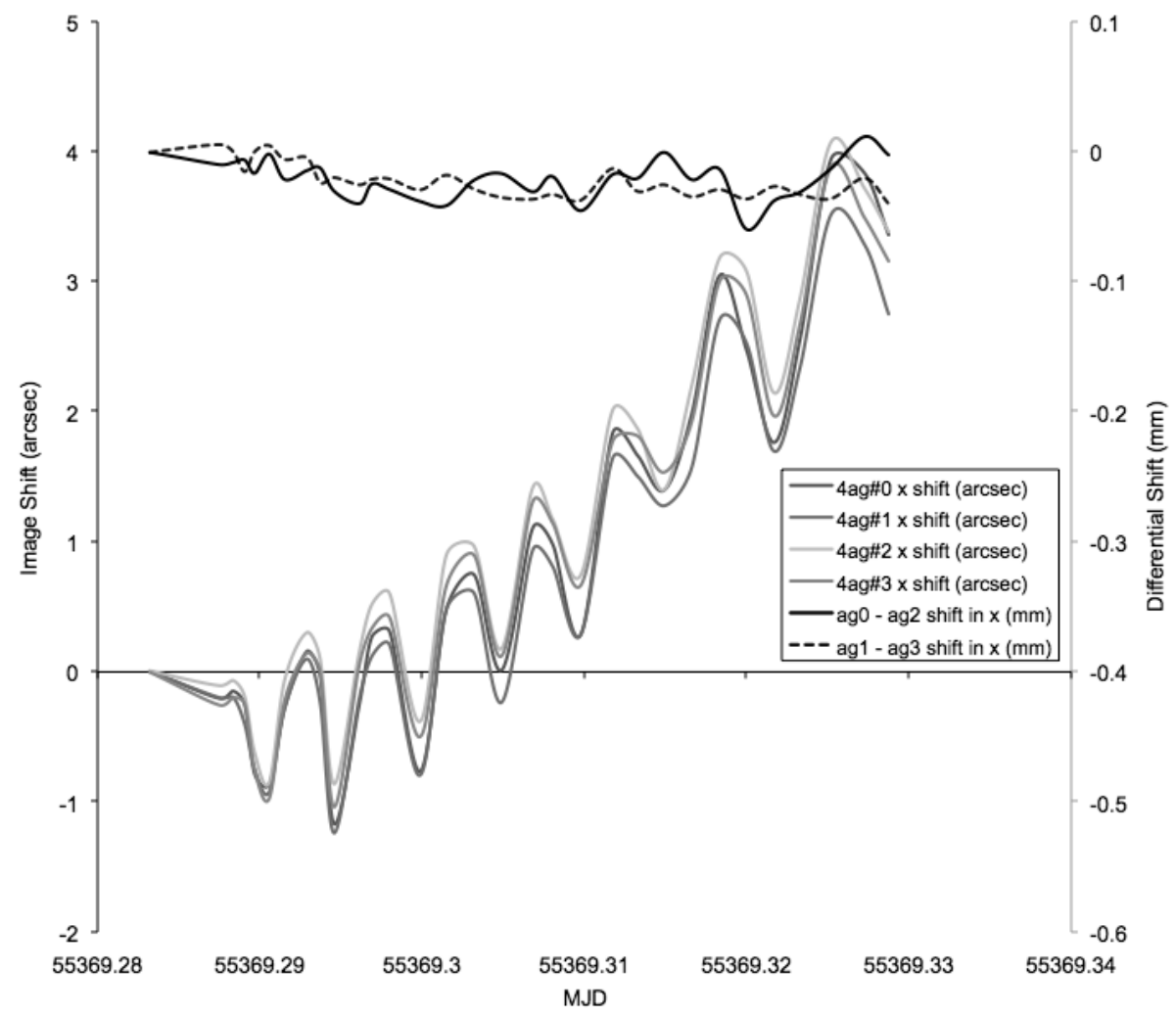

Figure 16. $\mathrm{x}$ image shift plot for each individual guiders.

The centroid drift in $\mathrm{x}$ and $\mathrm{y}$ show an overall drift along with some noise. The $\mathrm{x}$ plot has an interesting frequency that will be investigated as an artifact of the mount. The x plot of Figure 13 shows the periodic error corrected out in the theoretical guided plot. The are about 6 data points or 'guider corrections' per period so this movement is guided out, but when running normally the guiding frequency is four times as fast so the corrections would be even better.

The separations of the guide shifts from each of the cameras are of particular interest. The image shifts are calibrated from the first image beginning at zero then over the time each of the individual camera shifts show some separation from one another. The conceptual idea was to look at the separation of y components of the guide signals and use that to compute an angular error, but the differential of the x components can't be explained by a rotation. The thought that it might be differential deflection doesn't seem correct as a gradual drift would be expected and the two opposing camera differential deflections are not drifting or well correlated with one another (see black lines from Figure 16). The difference in the opposing cameras is a random movement that would point to an uncertainty error in the centroid calculations from image to image. 


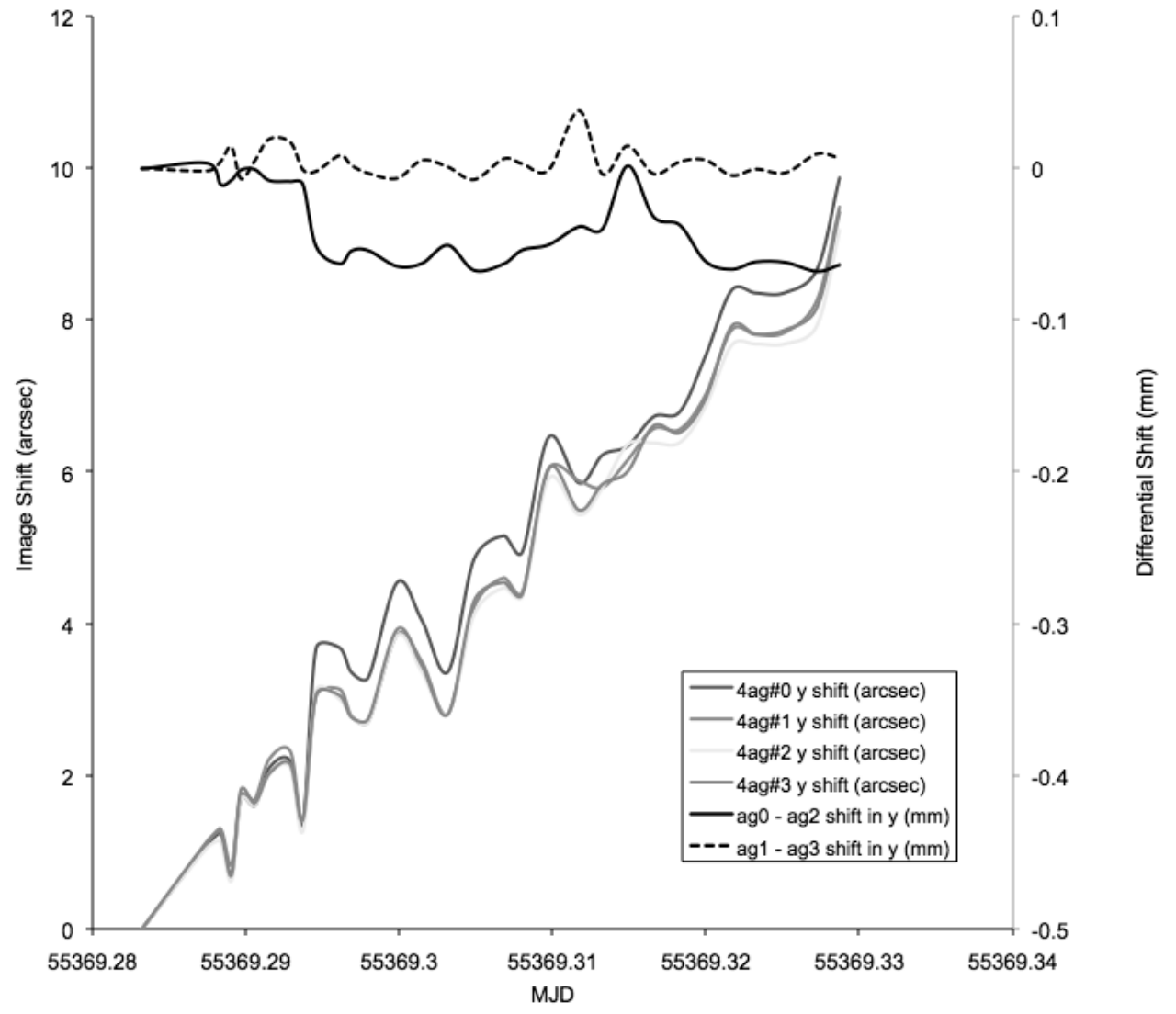

Figure 17. y image shift plot for each individual guiders.

The y plot for the individual camera doesn't show the even periodic translation that shows up in the $\mathrm{x}$ direction, but there is some movement back and forth while the telescope drifts in positive $\mathrm{y}$. The y direction can be picking up some of the translation seen in the $\mathrm{x}$ possibly due to the same mount error that is showing up very periodic in the $\mathrm{x}$ direction. Again the frequencies of these movements are slow enough that the guider can track them.

The differential shift in y between the opposing cameras shows a slightly higher magnitude than the $\mathrm{x}$, but again there is not a strong correlation between the two opposing differentials that would be expected for a rotation error. The assumed error in star centroids would be expected to be the same on y, but there is more differential on the y image shifts can be that can be investigated as rotation. 


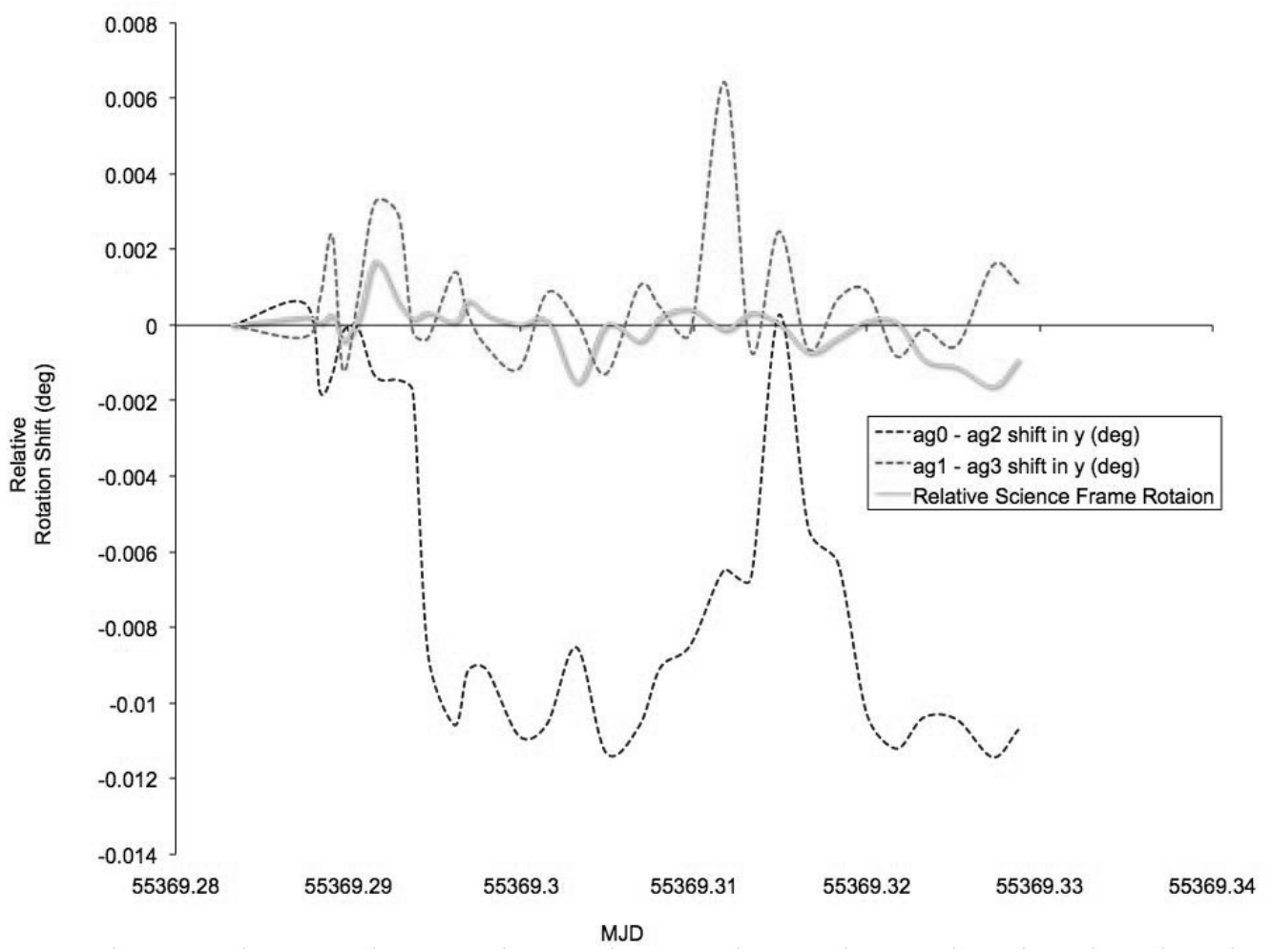

Figure 18. Relative rotation from differential movement in y image shifts compared to a WCS rotation fit for the science frames.

The differential shifts in y from Figure 17 are converted to degrees of rotation about the center of the science field. The WCS fit for the rotation of the science field is plotted in the solid light blue line. There is no correlation between the rotations found from the WCS fit and those calculated by the difference in y shifts. The magnitude of the calculated shifts are much greater than those from the WCS. The WCS fit was done on a dense field and would likely be more accurate than the rotation found using opposing guider centroids. The WCS fit rotations are small and account for movements on the guide chips less than .1 arc second. Very small movement of .1 arc second or less are not going to be able to resolved by the guide camera in order to correct.

\section{CONSLUSIONS AND FUTURE WORK}

- One of the guide cameras performs almost as good as the average of all for the $\mathrm{x}, \mathrm{y}$ corrections to the telescope pointing. Shorter exposures might benefit more from the averaging of all of the cameras.

- Actively guided long term exposures are void of long term drift and have scatter less than one arc second, which in most cases is providing guiding better than the seeing conditions.

- Differential movements in the $\mathrm{x}$ direction camera shifts are random and see to come from errors in determining the centroids and do not point to a differential deflection.

- Using the opposing guide cameras to calculate and correct for rotation didn't appear to give accurate results for this experiment.

- Some longer experiments with the telescope in different parts of the sky would help to confirm that these results are typical.

- Purposely rotating cassegrain during the experiment and looking at the accuracy of the calculated rotation from the autoguiders would determine if the rotation correction is a viable feature. 
- Upgrading the software to take images from all of the autoguiders automatically would allow much more data to easily be obtained.

- Investigate focus tracking.

\section{ACKNOWLADGEMENTS}

Joseph Tufts for conceptual design, testing, and support. Ben Haldeman for initial mechanical layout. Dave Jang for Blackfin and Sensor board design. Tim Lister for Figure 15. Eric Hawkins for software and testing support. Steve Foale for software development and diagnostics.

\section{REFERENCES}

[1] Booth, N. and Smith, A. S., [Infrared Detectors], Goodwin House Publishers, New York \& Boston, 241-248 (1997).

[2] Schempp, W.V., "CCD Camera for an autoguider", Proc. SPIE 1448, 129-133 (1991)

[3] Abbot, Timothy M.C., "An off-the-shelf autoguider for McDonald Observatory." Proc. SPIE 1235, 770-776 (1990).

[4] Why Self-Guiding is Essential 2003, Santa Barbara Instrument Group, Santa Barbara, viewed 22 June 2010 , < http://www.sbig.com/sbwhtmls/online.htm>

[5] EQASCOM \& AutoGuiding, The EQMOD Project, viewed 22 June 21, 2010, <http://eq-mod.sourceforge.net/docs/EQASCOM_Guiding.pdf> 